University of Nebraska - Lincoln

DigitalCommons@University of Nebraska - Lincoln

\title{
Lattice distortions and oxygen vacancies produced in Au+- irradiated nanocrystalline cubic zirconia
}

Philip D. Edmondson

Oak Ridge National Laboratory

William J. Weber

Oak Ridge National Laboratory, weberwj@ornl.gov

Fereydoon Namavar

University of Nebraska Medical Center

Yanwen Zhang

Oak Ridge National Laboratory, zhangy1@ornl.gov

Follow this and additional works at: https://digitalcommons.unl.edu/usdoepub

Edmondson, Philip D.; Weber, William J.; Namavar, Fereydoon; and Zhang, Yanwen, "Lattice distortions and oxygen vacancies produced in Au+-irradiated nanocrystalline cubic zirconia" (2011). US Department of Energy Publications. 356.

https://digitalcommons.unl.edu/usdoepub/356

This Article is brought to you for free and open access by the U.S. Department of Energy at DigitalCommons@University of Nebraska - Lincoln. It has been accepted for inclusion in US Department of Energy Publications by an authorized administrator of DigitalCommons@University of Nebraska - Lincoln. 


\title{
Lattice distortions and oxygen vacancies produced in $\mathrm{Au}^{+}$-irradiated nanocrystalline cubic zirconia
}

\author{
Philip D. Edmondson, ${ }^{a}$ William J. Weber, ${ }^{\mathrm{a}, \mathrm{b}}$ Fereydoon Namavar ${ }^{\mathrm{c}}$ and Yanwen Zhang, ${ }^{\mathrm{a}, \mathrm{b}, *}$ \\ ${ }^{a}$ Materials Science and Technology Division, Oak Ridge National Laboratory, Oak Ridge, TN 37831, USA \\ ${ }^{\mathrm{b}}$ Department of Materials Science \& Engineering, University of Tennessee, Knoxville, TN 37996, USA \\ ${ }^{\mathrm{c}}$ University of Nebraska Medical Center, Omaha, NE 68198, USA
}

Received 4 May 2011; revised 5 July 2011; accepted 5 July 2011

Available online 13 July 2011

The oxygen ion conductivity, attributed to an oxygen vacancy mechanism, of yttria-stabilized zirconia membranes used in solid oxide fuel cells is restricted due to trapping limitations. In this work, a high concentration of oxygen vacancies has been deliberately introduced into nanocrystalline stabilizer-free zirconia through ion-irradiation. Oxygen vacancies with different charge states can be produced by varying irradiation temperatures. Due to the reduced trapping sites and high oxygen vacancy concentration, this work suggests that the efficiency of solid oxide fuel cells can be improved.

Published by Elsevier Ltd. on behalf of Acta Materialia Inc.

Keywords: Nanocrystalline zirconia; Oxygen vacancy; Lattice spacing; Glancing incidence X-ray diffraction; Ion-beam modification

Yttria-stabilized zirconia is used as an oxygenion-conducting solid electrolyte in many devices, such as sensors and solid oxide fuel cells, due to its extraordinary oxygen transport properties, which are attributed to an oxygen vacancy mechanism [1]. The stabilization of zirconia with an aliovalent compound such as yttria $\left(\mathrm{Y}_{2} \mathrm{O}_{3}\right)$ serves a dual purpose in the system. First, pure zirconia exists in three different thermodynamically stable polymorphs at atmospheric pressure: a monoclinic phase at temperatures below $1400^{\circ} \mathrm{C}$, a tetragonal phase at temperatures of $1400-2600{ }^{\circ} \mathrm{C}$ and a cubic phase at temperatures above $2600{ }^{\circ} \mathrm{C}$. The cubic phase is the most interesting phase as an engineering material due to its isotropic expansion at various temperatures. By doping the zirconia with an aliovalent compound with concentrations of $\sim 8-10 \mathrm{~mol} . \%$ [1-5], the cubic phase of zirconia is stabilized at the temperatures typically experienced during the operation of oxygen sensors and fuel cells through the introduction of structural oxygen vacancies into the lattice. Secondly, the oxygen vacancies that serve to stabilize the zirconia also lead to the fast oxygen ion conductivity desired in many

\footnotetext{
* Corresponding author at: Materials Science and Technology Division, Oak Ridge National Laboratory, Oak Ridge, TN 37831, USA. Tel.: +1 865574 8518; fax: +1 865241 3650; e-mail: zhangy1@ ornl.gov
}

applications [2,3]. However, studies have shown that increase of the dopant concentration greater than $\sim 10$ mol. $\%$ reduces the ion conductivity [6,7]. This reduction in ion conductivity has been suggested to occur as a result of numerous mechanisms: phase transformation to the tetragonal phase of $\mathrm{ZrO}_{2}$, precipitation of ordered phases such as $\mathrm{Y}_{2} \mathrm{Zr}_{2} \mathrm{O}_{7}$, formation of microdomains, segregation of yttria to grain boundaries, and short-range ordering arising from the trapping of oxygen vacancies by the dopant cations [8]. Therefore the ion conductivity in stabilized zirconia films is maximized when doping with concentrations of $\sim 8-10 \mathrm{~mol} . \%$. It is therefore of technological interest to improve the oxygen ion conductivity in zirconia by introducing a high concentration of oxygen vacancies without the introduction of stabilizing elements.

Nanostructured materials, i.e. those having grain sizes well below $100 \mathrm{~nm}$, are leading a technological revolution. This is in part due to their exceptional size-dependent chemical, physical, optical and electrical properties compared to those of their micron-sized polycrystalline and single crystal counterparts [9-13]. Nanostructured materials are therefore attracting significant interest for use in a wide variety of applications, such as catalysts, advanced nuclear energy systems, solar cells, membranes, oxygen sensors and solid oxide fuel cells.

Recently, it has been demonstrated that it is possible to produce nanocrystalline tetragonal or cubic zirconia 
that is thermodynamically stable at atmospheric pressures without the addition of stabilizers $[10,13,14]$, i.e. they are nanostructurally stabilized. Furthermore, the grain size of pure cubic nanostructurally stabilized zirconia (NSZ) films can be tailored by varying the ion dose during energetic, heavy ion irradiation [14]. Moreover, a significant loss of oxygen is also reported, suggesting the production of oxygen vacancies as a result of the irradiation [14]. Thus it may be possible to tailor the oxygen vacancy content, and hence the oxygen ion conductivity, in cubic NSZ by ion irradiation that could lead to much higher ionic conductivity than that obtained in dopantstabilized zirconia films, where the conductivity is often limited by the interactions between the oxygen vacancies and the stabilizing elements, as described previously. This would serve to improve the reliability and heat management of solid oxide fuel cells, e.g. by enabling a lower operating temperature.

To date, most research has focused on radiation processes (primarily heavy ion irradiation) in either partially- or fully stabilized zirconia as single crystals or with micron-sized grains, or on nanograined $\mathrm{ZrO}_{2}$ that are either embedded in a foreign matrix or as free-standing particles [15-17]. The work presented here examines the effect of heavy ion irradiation on NSZ with a view to understanding the defect production, saturation and evolution of oxygen vacancies, and how this impacts the unit cell structure.

Thin films, approximately $300 \mathrm{~nm}$ thick, of NSZ in the cubic phase with initial grain sizes of 5-10 $\mathrm{nm}$ have been grown on top of a $\left(\begin{array}{lll}0 & 0 & 1\end{array}\right) \mathrm{Si}$ substrate using an ionbeam-assisted deposition technique [10]. These films have previously been shown to be structurally stable up to temperatures of $1123 \mathrm{~K}[5,10]$. The films were then irradiated with $2 \mathrm{MeV} \mathrm{Au}{ }^{+}$ions at a temperature of 160 or $400 \mathrm{~K}$ over a range of doses up to $\sim 35$ displacements per atom (dpa). The energy was chosen such that the energy deposited into the film was maximized whilst minimizing the number of ions implanted into the film. Subsequent to the irradiation, samples were analyzed using the complimentary techniques of glancing incidence X-ray diffraction (GIXRD) and cross-sectional transmission electron microscopy (XTEM). The GIXRD was performed using $\mathrm{Cu} K_{\alpha 1}$ X-rays. Specimens to be examined using XTEM

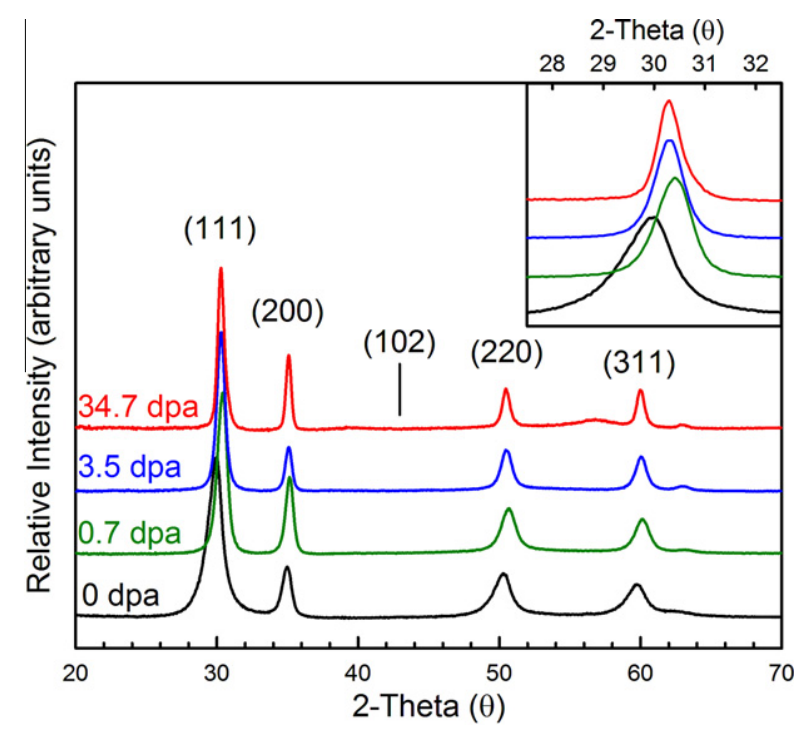

Figure 1. GIXRD spectra obtained for the as-deposited film and the films irradiated by $2 \mathrm{MeV} \mathrm{Au}$ at $400 \mathrm{~K}$. The irradiation doses and the diffraction indices, including the absent $\left(\begin{array}{lll}1 & 0 & 2\end{array}\right)$ index, are labelled. The inset shows a magnified view of the (llll 111$)$ peak.

were prepared by tripod polishing followed by ion milling to perforation using a Gatan PIPS. A JEOL 2010 transmission electron microscope operating at $200 \mathrm{keV}$ was used to image the specimens.

The curves shown in Figure 1 are the GIXRD results for the $400 \mathrm{~K}$ samples for the as-deposited $(0 \mathrm{dpa})$ sample, and those irradiated to doses of $0.7,3.5$ and $35 \mathrm{dpa}$. It can be seen that the as-deposited sample is the cubic polymorph. This is inferred by the absence of the (llll $\left.\begin{array}{lll}1 & 2\end{array}\right)$ reflection at $\sim 43^{\circ}$, which is characteristic of the tetragonal phase. As the dose is increased up to $35 \mathrm{dpa}$, there is no peak observed at $\sim 43^{\circ}$, indicating that the cubic phase is retained despite the significant energy deposition to the lattice during the irradiation. The absence of the tetragonal phase is confirmed by the selected area electron diffraction (SAED) patterns obtained during XTEM, as shown in Figure $2 \mathrm{a}$ and $\mathrm{b}$ for the as-deposited sample and the sample irradiated to $\sim 35 \mathrm{dpa}$ at $400 \mathrm{~K}$, respectively. The SAED patterns also indicate that there is a grain-coarsening effect occurring.
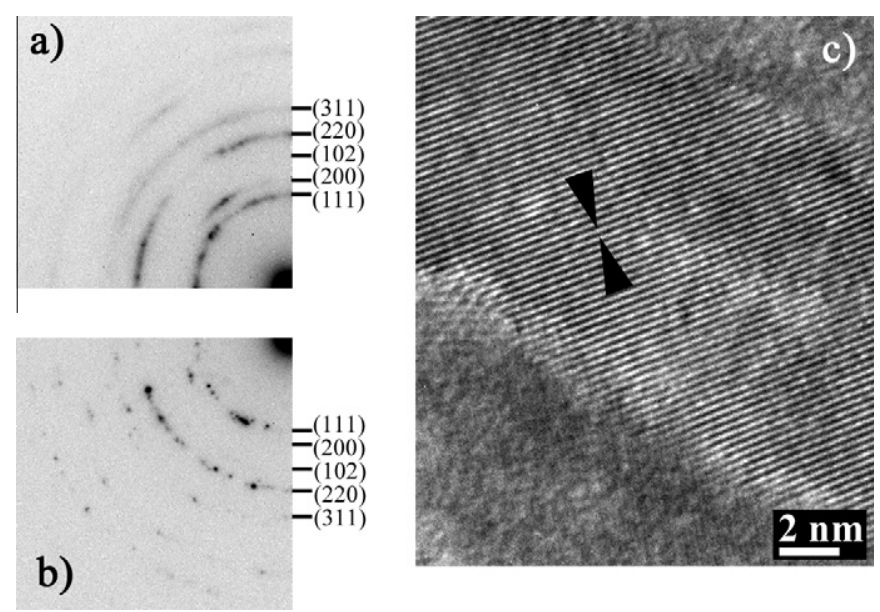

Figure 2. SAED patterns of (a) the as-deposited film and (b) the film irradiated by $2 \mathrm{MeV}$ Au to a dose of $\sim 35 \mathrm{dpa}$ at $400 \mathrm{~K}$. (c) A high-resolution TEM image of a typical grain in the as-deposited film. The arrows indicate a $d_{111}$ spacing of $2.51 \AA$. 
This is concluded from the transition of the diffuse SAED pattern in Figure 2a to a much sharper pattern with discrete diffraction spots becoming visible in Figure $2 \mathrm{~b}$. This grain-coarsening observation was attributed to a defect-stimulated grain growth mechanism [14]. The SAED patterns further indicate that the nanocrystalline grains are arranged in random orientations.

The inset shown in Figure 1 is a magnified view of the (1 111 l) peaks obtained by GIXRD. Two observations are immediately apparent: the narrowing of the peaks with increasing irradiation dose (dpa) and the shift in the positioning of the peaks. The narrowing of the peaks corresponds to the grain coarsening discussed earlier [14]. However, the shift in the peaks is related to a change in the spacing of the (1 111$)$ planes, $d_{111}$. This, in turn, is related to a change in the lattice parameter $a$ (proportional to $d_{111}$ by a factor of $\sqrt{ } 3$ ). The high-resolution micrograph shown in Figure $2 \mathrm{c}$ is of $\left(\begin{array}{lll}1 & 1 & 1\end{array}\right)$ lattice fringes from a typical grain in the as-deposited NSZ film. The lattice spacing indicated by the arrows is $2.51 \AA$, as calculated using a fast Fourier transform of the grain in the image. This is in close agreement with measurements obtained from the GIXRD (2.369 $)$ ), but is significantly lower than the theoretical value for cubic zirconia of $2.962 \AA$ [18]. GIXRD is a more accurate method for measuring lattice spacing than high-resolution TEM and allows more grains to be sampled.

Figure 3a shows the percentage difference between the $d_{111}$ spacings measured by GIXRD and the theoretical $d_{111}$ spacing as a function of dpa. Measurements of the higher order reflections gave the same results, but the (1 111 ) reflections are used here to allow for comparison with the TEM measurements. This result shows that, for the as-deposited film, there is a significant compression of the lattice from the ideal, represented by contraction of approximately $-20 \%$ from the theoretical $d_{111}$. This demonstrates that the lattice is highly stressed despite the use of Ar ions during the deposition process to relieve the stress [10]. The $400 \mathrm{~K}$ irradiation (open triangles and the dashed line) shows that there is a significant increase in the $d_{111}$ spacing compared to that of the as-deposited material of greater than $\sim 27 \%$ at low dpa. This indicates that there is a substantial amount of radiation induced stress relief in the system. Immediately following this stress relief phase, the $d_{111}$ spacing begins to reduce, before approaching a stable stress value of $\sim-2 \%$. A similar effect is observed for the $160 \mathrm{~K}$ irradiation (open squares, solid line), although the initial relaxation phase is not quite so dramatic as for the $400 \mathrm{~K}$ irradiation. Here the $d_{111}$ spacing, under $160 \mathrm{~K}$ irradiation, tends to stabilize at $\sim-9 \%$. The result in Figure $3 \mathrm{~b}$ shows the change in overall thickness of the film, measured by XTEM, as a function of dose. It is evident that the film thickness follows a similar trend to that of the $d_{111}$ spacing measurements. These correlated events indeed indicate that ion irradiation is an efficient tool to modify and control the lattice parameter throughout the film.

Previous studies have shown that $a$ may reduce significantly as a function of grain size for powdered nanocrystalline $\mathrm{CeO}_{2}$, particularly in the size range of the grains of interest here $(3-30 \mathrm{~nm})$ [19]. However, in the previous study, the effects of the unconstrained free surface may not be negligible. Moreover, the results shown (a)

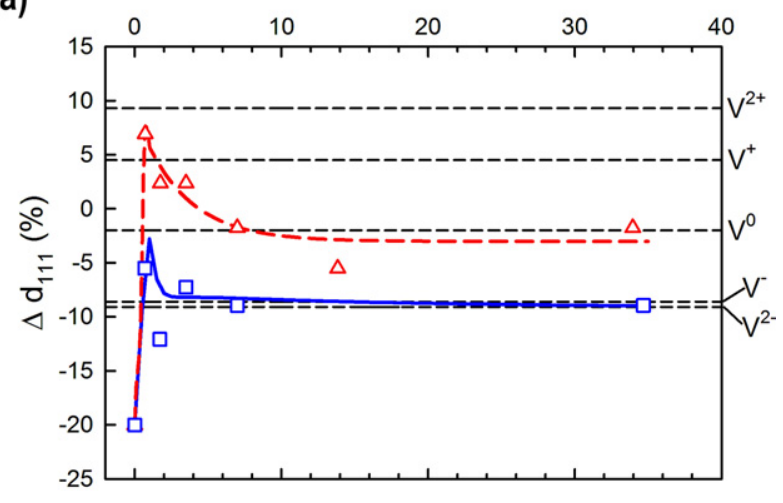

(b)

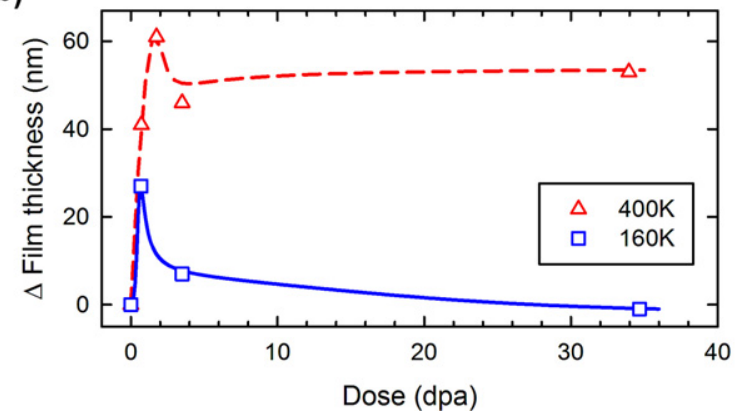

Figure 3. (a) A plot of the difference between the ideal $d_{111}$ spacing and that measured by GIXRD (as a percentage) as a function of dose. The distortions induced by oxygen vacancies with different charge states are overlaid. (b) A plot of the variation in film thickness measured by XTEM as a function of dose. The lines serve to guide the eye.

in Figure 4a, particularly for the $160 \mathrm{~K}$ irradiation, show that, whilst there is a relatively large increase in grain size of $\sim 6 \mathrm{~nm}$ (from 23.5 to $29.5 \mathrm{~nm}$ ) from 8 to $35 \mathrm{dpa}$, there is little, if any, change in $d_{111}$. This demonstrates that the variation in lattice parameter observed here is a result not of the change in the grain size, but of a different mechanism altogether. To understand the mechanism by which the lattice parameter changes, the types of defect, their accumulation and saturation in the NSZ films are evaluated. Whilst different configurations of defects may be formed in zirconia, such as the oxygen interstitials and anti-site defects, by far the most common defect is the oxygen vacancy. The oxygen divacancy has been observed [20], but only during swift heavy-ion irradiations performed at $8 \mathrm{~K}$. The graph shown in Figure $4 \mathrm{~b}$ shows that there is a significant loss of $\mathrm{O}$ (up to $\sim 19 \%$ ) as a result of the irradiation, and hence the most likely defect to exist in significant quantities is the isolated oxygen vacancies. An ab initio study of isolated oxygen vacancies in nanocrystalline cubic zirconia [18] has shown that five different charge states of the defect may exist: $\mathrm{V}^{2+}, \mathrm{V}^{+}, \mathrm{V}^{0}, \mathrm{~V}^{-}$and $\mathrm{V}^{2-}$. In the same study, it was shown that the production of an oxygen vacancy could result in a change in the atom-atom distances in the neighborhood of the defect, the amount of distortion being dependent on the charge state of the defect. The distortions are: $9.3 \%\left(\mathrm{~V}^{2+}\right), 4.5 \%\left(\mathrm{~V}^{+}\right)$, $-2.0 \%\left(\mathrm{~V}^{0}\right),-8.6 \%\left(\mathrm{~V}^{-}\right)$and $-9.1 \%\left(\mathrm{~V}^{2-}\right)$, with positive values representing a lattice expansion, negative values a lattice contraction. The positively and neutrally charged defects are symmetrical, whilst the negatively charged 
(a)

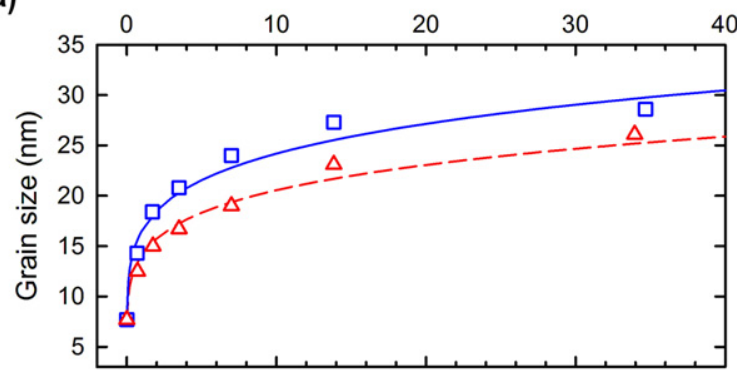

(b)

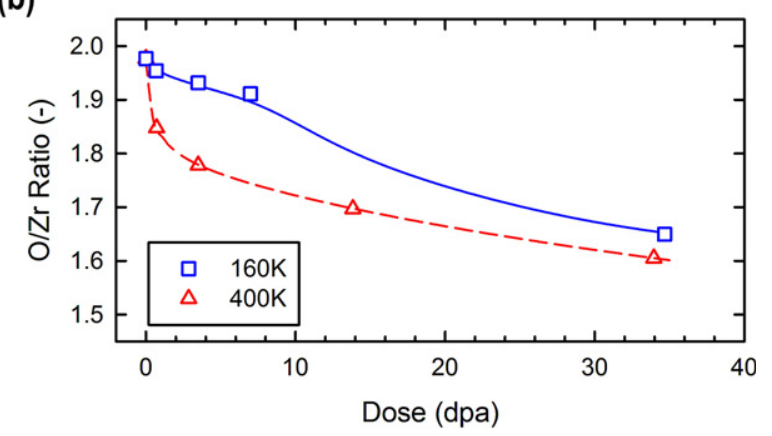

Figure 4. Plots of the grain size (a) and the $\mathrm{O} / \mathrm{Zr}$ ratio (b) as a function of dose for both the 160 and $400 \mathrm{~K}$ irradiations. The lines in all the plots are to serve as a guide to the eye.

vacancies are asymmetrical and hence the distortion stated above is for the maximum asymmetric displacement of the atom-atom distance.

In Figure 3a the distortions produced by the oxygen vacancies of the different charge states are overlaid. It can be seen that, as the vacancy defects begin to saturate within the nanocrystalline matrix or along the grain boundaries at an ion dose exceeding $10 \mathrm{dpa}$, three types of defects are the most likely to exist: $\mathrm{V}^{0}, \mathrm{~V}^{2-}$ and $\mathrm{V}^{-}$. For the $400 \mathrm{~K}$ irradiation, the $\mathrm{V}^{0}$ defect appears to be the dominant defect. For the $160 \mathrm{~K}$ irradiation, it is not quite so clear, with both the $\mathrm{V}^{2-}$ and $\mathrm{V}^{-}$defects producing similar distortions to the lattice; however, it suggests that these two defects may be the dominant defects at $160 \mathrm{~K}$. Electron paramagnetic resonance experiments [17] performed on $\gamma$-ray-irradiated monoclinic $\mathrm{ZrO}_{2}$ has shown that the $\mathrm{V}^{-}$defect is the most likely to exist, leading to the possibility that this is the most dominant defect at $160 \mathrm{~K}$. This cannot be categorically proven, however, as the difference in the crystal structures (monoclinic phase versus cubic phase) may lead to different charge states being dominant.

It is clear that there is a thermal component to the stability of the $\mathrm{V}^{0}, \mathrm{~V}^{2-}$ and $\mathrm{V}^{-}$defects. As the negative charge state vacancies are not dominant at the higher $400 \mathrm{~K}$ irradiation, this is an indication that, at some temperature between 160 and $400 \mathrm{~K}$, these defects may become mobile, leading to interaction and annihilation of the defects or neutralization to the $\mathrm{V}^{0}$ defect. The $\mathrm{V}^{0}$ defect is clearly thermodynamically stable at $400 \mathrm{~K}$.

In, conclusion it has been shown that there is a significant alteration to the lattice parameter resulting from heavy ion irradiation. The change in the lattice parameter, inferred by a change in the $d_{111}$ spacing, is due to the production and saturation of oxygen vacancies of differ- ent charge states, and the extent of the lattice distortion is dependent on the charge state of the oxygen vacancies produced. We have shown that, at $400 \mathrm{~K}$, the neutral charge state oxygen vacancy, $\mathrm{V}^{0}$, is the dominant defect, while at $160 \mathrm{~K}^{2}$ the $\mathrm{V}^{2-}$ and $\mathrm{V}^{-}$charge states may be the dominant defects. These results also show that the negatively charged oxygen vacancies are not thermodynamically stable at elevated temperatures, i.e. those above $400 \mathrm{~K}$.

This work was supported as part of the Materials Science of Actinides, an Energy Frontier Research Center funded by the US Department of Energy, Office of Basic Energy Sciences, Materials Sciences and Engineering Division. A portion of the research was performed at the Environmental Molecular Sciences Laboratory (EMSL), a national scientific user facility sponsored by the Department of Energy's Office of Biological and Environmental Research, and located at Pacific Northwest National Laboratory.

[1] U. Brossmann, R. Wurschum, U. Sodervall, H.-E. Schaefer, J. Appl. Phys. 85 (1999) 7646-7654.

[2] B.W. Busch, W.H. Schulte, E. Garfunkel, T. Gustafsson, W. Qi, R. Nieh, J. Lee, Phys. Rev. B 62 (2000) R13290.

[3] G. Knöner, K. Reimann, R. Röwer, U. Södervall, H.-E. Schaefer, Proc. Natl. Acad. Sci. USA 100 (2003) 3870-3873.

[4] R. Devanathan, W.J. Weber, S.C. Singhal, J.D. Gale, Solid State Ionics 177 (2006) 1251-1258.

[5] Y.L. Soo, P.J. Chen, S.H. Huang, T.J. Shiu, T.Y. Tsai, Y.H. Chow, Y.C. Lin, S.C. Weng, S.L. Chang, G. Wang, C.L. Cheung, R.F. Sabirianov, W.N. Mei, F. Namavar, H. Haider, K.L. Garvin, J.F. Lee, H.Y. Lee, P.P. Chu, J. Appl. Phys. 104 (2008) 113535-1-113535-5.

[6] A.I. Ioffe, D.S. Rutman, S.V. Karpachov, Electrochim. Acta 23 (1978) 141-142.

[7] S.P.S. Badwal, Solid State Ionics 52 (1992) 23-32.

[8] J. Kondoh, S. Kikuchi, Y. Tomii, Y. Ito, J. Electrochem. Soc. 145 (1998) 1536-1550.

[9] A.P. Alivisatos, Science 271 (1996) 933-937.

[10] F. Namavar, C.L. Cheung, R.F. Sabirianov, W.-N. Mei, X.C. Zeng, G. Wang, H. Haider, K.L. Garvin, Nano Lett. 8 (2008) 988-996.

[11] D.J. Norris, A.L. Efros, S.C. Erwin, Science 319 (2008) 1776-1779.

[12] M.V. Kovalenko, M. Scheele, D.V. Talapin, Science 324 (2009) 1417-1420.

[13] J. Lian, J. Zhang, F. Namavar, Y. Zhang, F. Lu, H. Haider, K. Garvin, W.J. Weber, R.C. Ewing, Nanotechnology 20 (2009) 245303.

[14] Y. Zhang, W. Jiang, C. Wang, F. Namavar, P.D. Edmondson, Z. Zhu, F. Gao, J. Lian, W.J. Weber, Phys. Rev. B 82 (2010) 184105.

[15] A. Benyagoub, Phys. Rev. B 72 (2005) 094114.

[16] D.G. Lamas, A.M. Rosso, M.S. Anzorena, A. Fernández, M.G. Bellino, M.D. Cabezas, N.E. Walsöe de Reca, A.F. Craievich, Scripta Mater. 55 (2006) 553-556.

[17] S. Wright, R.C. Barklie, J. Appl. Phys. 106 (2009) 103915-103917.

[18] D. Muñoz Ramo, P.V. Sushko, J.L. Gavartin, A.L. Shluger, Phys. Rev. B 78 (2008) 235432.

[19] S. Deshpande, S. Patil, S.V.N.T. Kuchibhatla, S. Seal, Appl. Phys. Lett. 87 (2005) 133113-1-133113-3.

[20] J.-M. Costantini, F. Beuneu, K. Schwartz, C. Trautmann, J. Phys.: Condens. Matter 22 (2010) 315402. 